\title{
An Open Experimental Platform for Cultivating Innovative Network Talents
}

\author{
Fang Fang \\ Network Engineering and Research Center, \\ South China Univ. of Technology, \\ Guangzhou, China, 510641 \\ ffang@scut.edu.cn
}

\author{
Luo Xinge* \\ Network Engineering and Research Center, \\ South China Univ. of Technology, \\ Guangzhou, China, 510641 \\ xluo@scut.edu.cn
}

\begin{abstract}
In the face of a new generation of Internet-evolving technologies and applications, universities need to cultivate innovative network talents. In order to promote the innovation of network technology, we established an open experimental teaching platform for cultivating innovative network talents. We bring together the resources of universities, research institutes and companies to cooperate and innovate and conclude that platform has a positive effect on the cultivation of network talents in our university.
\end{abstract}

Keywords-Experiment; Platform; network; Innovative; Open

\section{INTRODUCTION}

Openness and innovation are important symbols of the Internet age. The purpose of the open experimental platform is to integrate Internet experimental resources and form a platform for scientific and technological innovation with a wide range of influence. The platform can provide open services for other universities and provide support for student entrepreneurship. At the same time, through cooperation with enterprises, an industry alliance is formed to promote the transformation of scientific research achievements.

\section{THE SIGNIFICANCE OF UNIVERSITY LABORATORY CONSTRUCTION}

\section{A. Research projects must be fully researched and explored} in the laboratory.

University laboratories are important places for teaching, research, discipline construction and social services. Only through laboratory innovation and verification can technology's advantages be transformed into productivity.

\section{B. University laboratories are an important means to further strengthen students' quality education and practical ability training.}

For a long time, if the teaching mode does not attach importance to practice, students will lack creativity and lack the ability to analyze and solve problems. Through experimental teaching in the laboratory, students are the main body and teachers guide them. The laboratory can bring more free space to students, improve their interest in learning and initiative, and cultivate students' overall quality and practical ability.

\section{University laboratories are an important base for experimental teaching and scientific research.}

The combination of experimental teaching and scientific research is an important way to cultivate innovative talents. This is the key to building an innovative talent training system. This is also an important way to improve students' spirit of innovation and their ability to promote individual development.

\section{Training talents with scientific research quality and innovative ability is an important goal of university laboratories.}

University laboratories are an important way to cultivate innovative talents and an important indicator of the university's strength and the quality of talents training.

\section{NEW TRENDS IN THE CONSTRUCTION OF INTERNET EXPERIMENTAL PLATFORMS IN FOREIGN UNIVERSITIES}

\section{A. New Trends in the Construction of Internet Experimental} Platforms in Foreign Universities

The global application and promotion of the Internet has made people feel the convenience brought by the Internet. The Internet has become an important part of human social activities in areas such as business, scientific research and healthcare. Before the Internet was officially commercialized, its applications were born in the laboratory.

Foreign universities have always attached importance to the construction of experimental platforms and are committed to the next generation of Internet applications and technologies.

Experimental platforms initiated by the University Alliance, such as GENI (Global Environment for Network Innovations), collaborate in laboratories through virtualization to jointly undertake research on future network technologies and strive to take the lead in the next generation of Internet technology applications. 
B. The Status of the Current Network Experiment Platform in Chinese Universities

- The "985, 211 Project" promoted about 65\% of universities to build network basic laboratories.

- $50 \%$ of universities have established network engineering labs based on application verification.

- About $30 \%$ of universities have established professional laboratories that differ in their disciplines. About $30 \%$ of universities have established professional laboratories in different disciplines, such as information security, grid technology, mobile computing, network management and performance, analysis and testing, IP voice and video, distributed technology, and P2P.

- $\quad 7.9 \%$ of universities have set up research-oriented key laboratories for technological innovation and applied research.

In general, the construction of network technology laboratories in universities in our country started relatively late, and there is a big gap with foreign universities in the construction of experimental platforms. To a certain extent, this will form a constraint on China's future Internet technology and applications, and thus also affect China's next-generation Internet technology innovation, application promotion and personnel training strategy. [2]

\section{The Network Experiment Platform in Universities Faces Improvement and Development}

1) Improve lab utilization, implement inter-school sharing programs, and participate in social service systems.

Open laboratories increase the utilization rate of laboratories, solve vacancy and waste of laboratory time and equipment, make up for lack of local experimental resources, and promote the cooperation between universities in teaching and research.

2) Industry-university-research cooperation:

complementary advantages, strong alliances, academic exchanges, scientific research collaboration

Industry-university-research cooperation can realize university network laboratory resource sharing, cooperation innovation and research, jointly undertake scientific research projects, jointly develop public services, promote regional economic development, and realize the transformation of scientific research results. This is the common task of the construction of university laboratory platforms and the core of the development of laboratory construction.

Integration, sharing, and innovation are trends in the current development of network labs. The future network experimental platform can not only carry out normal teaching experiments, but also carry out academic research and application experiments, and it is also a platform for scientific research cooperation and academic exchange with domestic universities, international organizations, and international universities.

\section{THE DESIGN OF OPEN EXPERIMENTAL PlATFORM}

\section{A. The Characteristics of Open Experimental Platform}

- Forward-looking: The technical scope of the experimental platform covers the future network, cloud computing, and the Internet of Things. Under limited financial conditions, we have built a comprehensive experimental platform that adapts to the development of computer network technology and integrates teaching, training, and social services.

- Cross-cutting: The construction of the experimental platform involves multiple technical fields, and there is a certain degree of relevance between the technical directions. SDN (Software Defined Network) and NFV (Network Function Virtualization) technologies bring higher stability and lower costs to the network. This experimental platform can facilitate cross-disciplinary research and practice in different disciplines.

- Openness: All functions of the platform provide open interfaces to external parties. After studying in depth, teachers and students can exert their own innovation and carry out secondary development.

- Savings: Experiments in multiple scientific research directions share the same set of physical resources and do not need to build experimental resources independently. The experimental platform can achieve on-demand allocation of resources, flexible scheduling, and scalable services.

\section{B. Open Design Platform Design Overview}

\section{1) SDN/NFV network architecture}

As a new type of network architecture, SDN/NFV advocates software and virtualization, which can completely break through the existing network dilemma. It has become an important trend and feature of future network evolution. Relevant technologies and protocols will be used to constitute Future network infrastructure.

SDN can effectively improve the efficiency of resource use across the entire network, improve network virtualization capabilities. The centralized control layer can complete functions such as route estimation, centralized resource monitoring, and topology management. It can accurately collect resource usage of the entire network and safely isolate the virtual network between different users. The application layer provides a programmable environment by opening rich interfaces and virtual networks. The configured capabilities are open to end users and meet the needs of users to adjust the network as needed [1].

NFV uses virtualization technology to decouple traditional communication device functions from hardware, and uses common computing, storage, and network devices to implement communication network functions, breaking the limitations of proprietary hardware on the network. NFV helps improve the efficiency of network construction, management, and maintenance. Under the NFV approach, the on-line and update of new services are transformed from traditional hardware construction and cutover to software loading, and the 
construction period is greatly shortened. Combined with the scale advantage of the cloud computing resource pool, it can realize a variety of business sharing resources and centralized management, greatly improving management and maintenance efficiency [1].

2) Platform Management Diagram

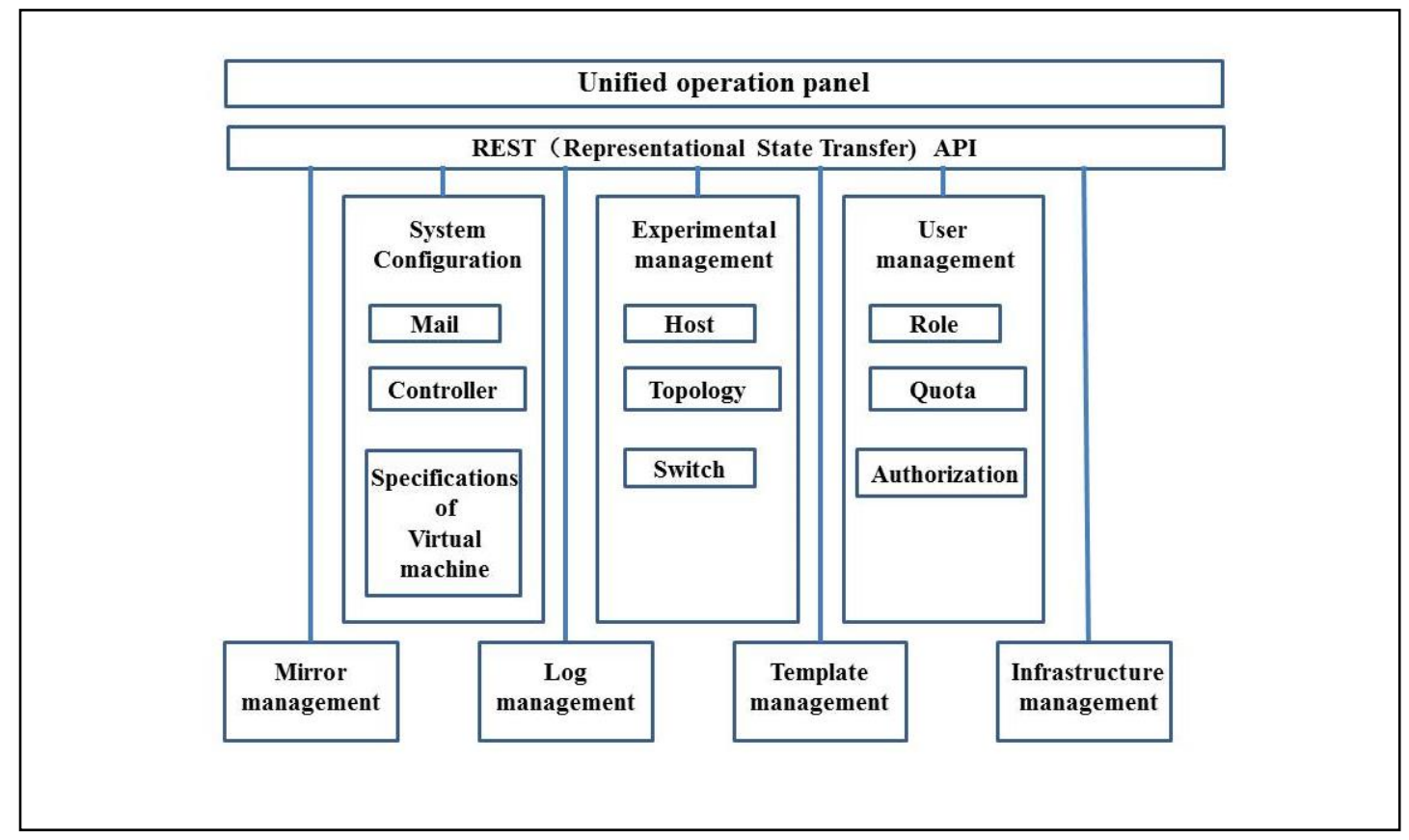

Fig. 1. Platform Management Diagram

3) Experimental platform functional design

The experimental platform can virtualize a highly customized virtual network topology environment with a limited infrastructure to accommodate the needs of different network experiments. The open experiment platform has the following main functions:

- The platform provides management of the network topology: Users can add or remove controllers, virtual switches, virtual hosts, virtual gateways, and virtual links, and customize their own network topology.

- The platform provides management of virtual nodes: virtual nodes include controllers, virtual switches, virtual hosts, and virtual gateways. The status and configuration of these resources need to be maintained.

- The platform provides management of virtual links in the network, including management of link status and configuration information.

- The platform provides monitoring of virtual nodes and virtual links.

- The platform provides one-click creation and template management functions.

- vController provides support for multiple SDN open source controllers and commercial SDN controllers.

- vGateway provides NFV capabilities to support open source NFV facilities and commercial NFV facilities.

\section{4) Experimental topology}

Take the example of building an SDN experiment network. The network includes an experiment controller, three virtual OVS (Open vSwitch) switches, a virtual host, and a virtual gateway. The virtual gateway provides DHCP services and can access external networks. The experimental topology is as shown below:

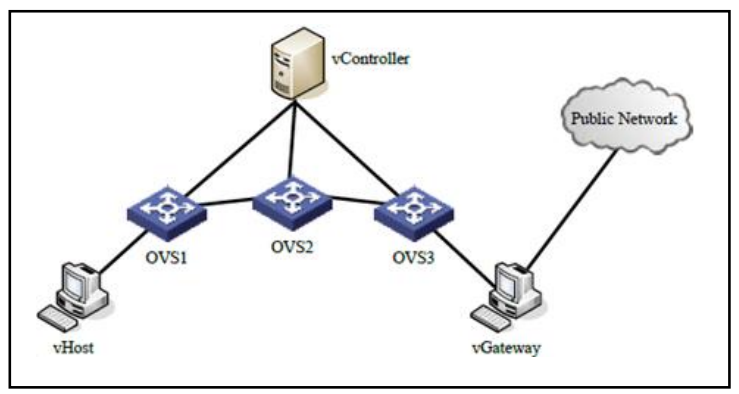

Fig. 2. Example of experimental network topology

For the above experimental topology requirements, based on the OpenStack virtualization technology, the open experiment platform implements user requirements from the following points:

- Virtual nodes: based on OpenStack compute virtualization capabilities, implement experimental controllers, virtual switches, virtual hosts, and virtual gateways; 
- Virtual link: Based on the OpenStack network virtualization function, establish a network data flat and control flat communication links;

- Experimental topology: A highly customized experimental topology is achieved through the combination of virtual nodes and virtual links.

According to the OpenStack virtualization implementation method and the business logic of the open experiment platform, the basic requirements of the open experiment platform are designed one by one. Through the combination of functions, a highly customized network environment is realized to meet the experimental requirements of different scenarios.

The experiment environment creation process: creating control flat links, creating data flat links, and creating virtual nodes.

\section{5) Virtual Router Software}

The virtual router software provides advanced routing functionality but does not reduce the reliability and performance of the hardware network connection scheme. Virtual routers provide firewall and VPN capabilities through high-performance software that provides advanced routing capabilities and security in physical, virtual, and cloud networking environments. Virtual routers build advanced multi-layered networks in a virtualized environment: Add, configure, and move network services on demand.

The virtual routers provided with the open experimental platform can easily be expanded and fully support IPv4 and IPv6 advanced unicast and multicast routing protocols. IPsec and OpenSSL support inter-site and remote access VPNs with powerful IPv4 and IPv6 firewall capabilities.

\section{The Innovations of Open Experiment Platform}

- Quickly build multiple types of network environments based on templates;

- Users can customize multiple types of network topology;

- Can simulate network traffic optimization between cross-domain data centers;

- Teachers can use the experimental platform to customize the experimental course.

\section{APPLICATION OF SDN IN CAMPUS NETWORK}

The experimental results based on the open experimental platform can be applied in the university campus network.

\section{A. SDN is used in data centers and cloud computing environments}

Routes are dynamically scheduled according to network load without affecting performance. Therefore, some network devices can be selectively shut down or suspended to enter power-saving mode when the network load is not high.

Current data center networks often perform virtual machine migration, dynamic changes in load balancing mechanisms, switch firmware upgrades, switch failure recovery, and access to new switches. These changes must be carefully deployed to avoid affecting the internal operations of the data center. The biggest challenge in this type of business is to synchronize the load of the link with the changes or congestion.

The SDN architecture brings an opportunity to solve this problem, which can reduce the demand for network planning to ensure the smooth operation of the data center network during software updates. With the development of mobile Internet and cloud computing technologies, more and more computing and data are being moved to the cloud. The communication bandwidth between data centers is very expensive and the demand is huge. The traditional network cannot adapt itself intelligently. The SDN controller can monitor the traffic and intelligently adapt the switch to network behavior to meet the traffic demand of the data center server [3].

\section{B. In addition to its potential in data center networks, SDN has many applications in the management and security of campus networks.}

Virtual network partitioning and mobility management can simplify the network management and control methods. The network dynamic access control system implements finegrained distributed security access functions by deploying security policies and distributed monitoring systems at high levels. Network virtualization can divide the same physical network into multiple virtual slices. Each slice is isolated but the physical devices are not isolated. Each virtual network controller has its own topology view to achieve the maximum and optimal use of the physical network [3].

\section{CONCLUSION}

South China University of Technology keeps up with the new trend of network development and actively participates in the application and research of future network technologies. In order to better serve the university teachers and students and improve the influence of the university in related fields, we built this professional open experimental platform. The experimental platform has been used for the training of SDN innovation technology in Universities, and it is also the official training and competition platform for the SDN application innovation contest.

\section{REFERENCES}

[1] https://www.sdnlab.com/, 2018

[2] http://www.dragonlab.org/, 2018

[3] Huang tao, Liu jiang, Wei liang, Zhang jiao, Liu yunjie, Softwaredefined network core principle and application practice, POSTS\&TELECOM PRESS,2014,pp190-191. (In Chinese) 